\title{
Beyond WIMPs: the Quark (Anti) Nugget Dark Matter
}

\author{
Ariel Zhitnitsky ${ }^{1, a}$ \\ ${ }^{1}$ Department of Physics and Astronomy, University of British Columbia, Vancouver, Canada
}

\begin{abstract}
We review a testable dark matter (DM) model outside of the standard WIMP paradigm. The model is unique in a sense that the observed ratio $\Omega_{\text {dark }} \simeq \Omega_{\text {visible }}$ for visible and dark matter densities finds its natural explanation as a result of their common QCD origin when both types of matter (DM and visible) are formed during the QCD phase transition and both are proportional to single dimensional parameter of the system, $\Lambda_{\mathrm{QCD}}$. We argue that the charge separation effect also inevitably occurs during the same QCD phase transition in the presence of the $C \mathcal{P}$ odd axion field $a(x)$. It leads to preferential formation of one species of nuggets on the scales of the visible Universe where the axion field $a(x)$ is coherent. A natural outcome of this preferential evolution is that only one type of the visible baryons (not anti- baryons) remain in the system after the nuggets complete their formation. Unlike conventional WIMP dark matter candidates, the nuggets and anti-nuggets are strongly interacting but macroscopically large objects. The rare events of annihilation of the anti-nuggets with visible matter lead to a number of observable effects. We argue that the relative intensities for a number of measured excesses of emission from the centre of galaxy (covering more than 11 orders of magnitude) are determined by standard and well established physics. At the same time the absolute intensity of emission is determined by a single new fundamental parameter of the theory, the axion mass, $10^{-6} \mathrm{eV} \lesssim m_{a} \lesssim 10^{-3} \mathrm{eV}$. Finally, we comment on implications of these studies for the axion search experiments, including microwave cavity and the Orpheus experiments.
\end{abstract}

\section{Introduction}

This talk is mostly based on recent paper [1]. It is generally assumed that the Universe began in a symmetric state with zero global baryonic charge and later, through some baryon number violating process, evolved into a state with a net positive baryon number. As an alternative to this scenario we advocate a model in which "baryogenesis" is actually a charge separation process in which the global baryon number of the Universe remains zero. In this model the unobserved antibaryons come to comprise the dark matter in form of the dense heavy nuggets, similar to the Witten's strangelets [2]. Both quarks and antiquarks are thermally abundant in the primordial plasma but, in addition to forming conventional baryons, some fraction of them are bound into heavy nuggets of quark matter. Nuggets of both matter and antimatter are formed as a result of the dynamics of the axion domain walls $[1,3,4]$, some details of this process will be discussed later in the text.

ae-mail: arz@phas.ubc.ca 
An overall coherent baryon asymmetry in the entire Universe is a result of the strong $\mathrm{CP}$ violation due to the fundamental $\theta$ parameter in QCD which is assumed to be nonzero at the beginning of the QCD phase transition. This source of strong CP violation is no longer available at the present epoch as a result of the axion dynamics, see original [5-7] and more recent papers [8-15] on the subject. Were $\mathrm{CP}$ symmetry to be exactly preserved an equal number of matter and antimatter nuggets would form resulting in no net "baryogenesis". However, CP violating processes associated with the axion $\theta(x)$ term in QCD result in the preferential formation of one type of species. This preference is essentially determined by the dynamics of coherent axion field $\theta(x)$ at the initial stage of the nugget's formation.

Asymmetric production of the nuggets directly translates into asymmetry between visible baryons and anti-baryons because the total baryon charge is a conserved quantity in this framework. If more anti-nuggets are produced in the system then less conventional anti-baryons remain in the system. These remaining anti-baryons in the plasma then annihilate away leaving only the baryons whose antimatter counterparts are bound in the excess of anti-nuggets and thus unavailable to annihilate. One should emphasize that the resulting asymmetry is order of one. It is not sensitive to a relatively small magnitude of the axion field, irrespectively to the magnitude of $\theta(x) \sim\left(10^{-2}-10^{-4}\right)$ at the beginning of the QCD phase transition as long as it remains coherent on the scale of the Universe, see [1] for the details. This is precisely the main reason of why the visible and dark matter densities must be the same order of magnitude

$$
\Omega_{\mathrm{dark}} \approx \Omega_{\mathrm{visible}}
$$

as they both proportional to the same fundamental $\Lambda_{\mathrm{QCD}}$ scale, and they both are originated at the same QCD epoch. In particular, if one assumes that the nuggets and anti-nuggets saturate the dark matter density today than the observed matter to dark matter ratio $\Omega_{\text {dark }} \simeq 5 \cdot \Omega_{\text {visible }}$ corresponds to a specific proportion when number of anti-nuggets is larger than number of nuggets by a factor of $\sim 3 / 2$ at the end of nugget's formation. This would result in a matter content with baryons, quark nuggets and antiquark nuggets in an approximate ratio

$$
\left|B_{\text {visible }}\right|:\left|B_{\text {nuggets }}\right|:\left|B_{\text {antinuggets }}\right| \simeq 1: 2: 3,
$$

with no net baryonic charge. If these processes are not fundamentally related the two components $\Omega_{\text {dark }}$ and $\Omega_{\text {visible }}$ could easily exist at vastly different scales.

Unlike conventional dark matter candidates, dark-matter/antimatter nuggets are strongly interacting but macroscopically large. They do not contradict the many known observational constraints on dark matter or antimatter for three main reasons [16]:

- They carry a huge (anti)baryon charge $|B| \gtrsim 10^{25}$, and so have an extremely tiny number density;

- The nuggets have nuclear densities, so their effective interaction is small $\sigma / M \sim 10^{-10} \mathrm{~cm}^{2} / \mathrm{g}$, well below the typical astrophysical and cosmological limits which are on the order of $\sigma / M<1 \mathrm{~cm}^{2} / \mathrm{g}$;

- They have a large binding energy such that the baryon charge in the nuggets is not available to participate in big bang nucleosynthesis (BвN) at $T \approx 1 \mathrm{MeV}$.

To reiterate: the weakness of the visible-dark matter interaction in this model due to the small geometrical parameter $\sigma / M \sim B^{-1 / 3}$ rather than due to the weak coupling of a new fundamental field to standard model particles. It is this small effective interaction $\sim \sigma / M \sim B^{-1 / 3}$ which replaces the conventional requirement of sufficiently weak interactions for WIMPs.

A fundamental measure of the scale of baryogenesis is the baryon to entropy ratio at the present time

$$
\eta \equiv \frac{n_{B}-n_{\bar{B}}}{n_{\gamma}} \simeq \frac{n_{B}}{n_{\gamma}} \sim 10^{-10}
$$


If the nuggets were not present after the phase transition the conventional baryons and anti-baryons would continue to annihilate each other until the temperature reaches $T \simeq 22 \mathrm{MeV}$ when density would be 9 orders of magnitude smaller than observed. This annihilation catastrophe, normally thought to be resolved as a result of "baryogenesis," is avoided in our proposal because more antibaryon charges than baryon charges are hidden in the form of the macroscopical nuggets and thus no longer available for annihilation. Only the visible baryons (not anti-baryons) remain in the system after nugget formation is fully completed.

In our proposal (in contrast with conventional models) the ratio $\eta$ is determined by the formation temperature $T_{\text {form }}$ at which the nuggets and anti-nuggets basically have completed their formation and below which annihilation with surrounding matter becomes negligible. This temperature is determined by many factors: transmission/reflection coefficients, evolution of the nuggets, expansion of the universe, cooling rates, evaporation rates, the dynamics of the axion domain wall network, etc. In general, all of these effects will contribute equally to determining $T_{\text {form }}$ at the QCD scale. Technically, the corresponding effects are hard to compute as even basic properties of the QCD phase diagram at nonzero $\theta$ are still unknown. However, an approximate estimate of $T_{\text {form }}$ is quite simple as it must be

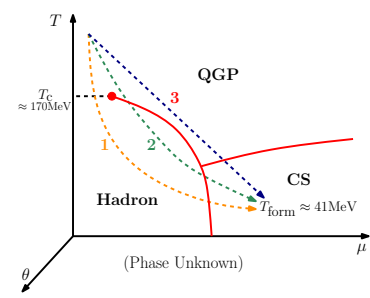

Figure 1. The conjectured phase diagram. The plot is taken from [1].

Possible cooling paths are denoted as path 1,2 or 3 . The phase diagram is in fact much more complicated as the dependence on the third essential parameter, the $\theta$ is not shown as it is largely unknown. It is assumed that the final destination after the nuggets are formed is the CS region with $T_{\text {form }} \approx 41$ $\mathrm{MeV}, \mu>\mu_{c}$ and $\theta \approx 0$, corresponding to the presently observed ratio (3), see text for the details.

expressed in terms of the gap $\Delta \sim 100 \mathrm{MeV}$ when the colour superconducting phase sets in inside the nuggets. The observed ratio (3) corresponds to $T_{\text {form }} \simeq 41 \mathrm{MeV}$ which is indeed a typical QCD scale slightly below the critical temperature $T_{c} \simeq 0.6 \Delta$ when colour superconductivity (CS) sets in. In different words, in this proposal the ratio (3) emerges as a result of the QCD dynamics when process of charge separation stops at $T_{\text {form }} \simeq 41 \mathrm{MeV}$, rather than a result of baryogenesis when a net baryonic charge is produced.

\section{Quark (anti) nugget DM confronting the observations}

While the observable consequences of this model are on average strongly suppressed by the low number density of the quark nuggets $\sim B^{-1 / 3}$ as explained above, the interaction of these objects with the visible matter of the galaxy will necessarily produce observable effects. Any such consequences will be largest where the densities of both visible and dark matter are largest such as in the core of the galaxy or the early universe. In other words, the nuggets behave as a conventional cold DM in the environment where density of the visible matter is small, while they become interacting and emitting radiation objects (i.e. effectively become visible matter) when they are placed in the environment with sufficiently large density.

The relevant phenomenological features of the resulting nuggets are determined by properties of the so-called electro-sphere as discussed in original refs. [17-22]. These properties are in principle, calculable from first principles using only the well established and known properties of QCD and QED. As such the model contains no tunable fundamental parameters, except for a single mean baryon number $\langle B\rangle$ which itself is determined by the axion mass $m_{a}$ as we already mentioned.

A comparison between emissions with drastically different frequencies from the centre of galaxy is possible because the rate of annihilation events (between visible matter and antimatter DM nuggets) 
is proportional to the product of the local visible and DM distributions at the annihilation site. The observed fluxes for different emissions thus depend through one and the same line-of-sight integral

$$
\Phi \sim R^{2} \int d \Omega d l\left[n_{\mathrm{visible}}(l) \cdot n_{D M}(l)\right]
$$

where $R \sim B^{1 / 3}$ is a typical size of the nugget which determines the effective cross section of interaction between DM and visible matter. As $n_{D M} \sim B^{-1}$ the effective interaction is strongly suppressed $\sim B^{-1 / 3}$ as we already mentioned in the Introduction. The parameter $\langle B\rangle \sim 10^{25}$ was fixed in this proposal by assuming that this mechanism saturates the observed $511 \mathrm{keV}$ line $[17,18]$, which resulted from annihilation of the electrons from visible matter and positrons from anti-nuggets. It has been also assumed that the observed dark matter density is saturated by the nuggets and anti-nuggets. It corresponds to an average baryon charge $\langle B\rangle \sim 10^{25}$ for typical density distributions $n_{\mathrm{visible}}(r), n_{D M}(r)$ entering (4). Other emissions from different bands are expressed in terms of the same integral (4), and therefore, the relative intensities are completely determined by internal structure of the nuggets which is described by conventional nuclear physics and basic QED. We present a short overview of these results below.

Some galactic electrons are able to penetrate to a sufficiently large depth of the anti-nuggets. These events no longer produce the characteristic positronium decay spectrum $(511 \mathrm{keV}$ line with a typical width of order $\sim$ few $\mathrm{keV}$ accompanied by the conventional continuum due to $3 \gamma$ decay) but a direct non-resonance $e^{-} e^{+} \rightarrow 2 \gamma$ emission spectrum. The transition between the resonance positronium decays and non-resonance regime is determined by conventional physics and allows us to compute the strength and spectrum of the $\mathrm{MeV}$ scale emissions relative to that of the $511 \mathrm{keV}$ line $[19,20]$. Observations by the Comptel satellite indeed show some excess above the galactic background consistent with our estimates.

Galactic protons incident on the anti-nugget will penetrate some distance into the quark matter before annihilating into hadronic jets. This process results in the emission of Bremsstrahlung photons at $\mathrm{x}$-ray energies [21]. Observations by the CHANDRA observatory apparently indicate an excess in $\mathrm{x}-$ ray emissions from the galactic centre. Hadronic jets produced deeper in the nugget or emitted in the downward direction will be completely absorbed. They eventually emit thermal photons with radio frequencies [22-24]. Again the relative scales of these emissions may be estimated and is found to be in agreement with observations.

We conclude this brief overview on observational constraints of the model with the following remark. This model which has a single fundamental parameter (the mean baryon number of a nugget $\langle B\rangle \sim 10^{25}$, corresponding to the axion mass $m_{a} \simeq 10^{-4} \mathrm{eV}$ ), and which enters all the computations is consistent with all known astrophysical, cosmological, satellite and ground based constraints as highlighted above. Furthermore, in a number of cases the predictions of the model are very close to the presently available limits, and very modest improving of those constraints may lead to a discovery of the nuggets. Even more than that: there is a number of frequency bands where some excess of emission was observed, and this model may explain some portion, or even entire excess of the observed radiation in these frequency bands.

\section{Five crucial ingredients of the proposal.}

In this section we explain the crucial elements of the proposal. The detail discussions for each ingredient can be found in original paper [1]. 


\section{1 $N_{D W}=1$ domain walls}

First important element of this proposal is the presence of the topological objects, the axion domain walls [25]. As we already mentioned the $\theta$ parameter is the angular variable, and therefore supports various types of the domain walls, including the so-called $N_{D W}=1$ domain walls when $\theta$ interpolates between one and the same physical vacuum state with the same energy $\theta \rightarrow \theta+2 \pi n$. The axion domain walls may form at the same moment when the axion potential get tilted, i.e. at the moment $T_{a}$ when the axion field starts to roll due to the misalignment mechanism. The tilt becomes much more pronounced at the phase transition when the chiral condensate forms at $T_{c}$. In general one should expect that the $N_{D W}=1$ domain walls form once the axion potential is sufficiently tilted, i.e. anywhere between $T_{a}$ and $T_{c}$.

One should comment here that it is normally assumed that for the topological defects to be formed the Peccei-Quinn (PQ) phase transition must occur after inflation. This argument is valid for a generic type of domain walls with $N_{D W} \neq 1$. The conventional argument is based on the fact that few physically different vacua with the same energy must be present inside of the same horizon for the domain walls to be formed. The $N_{D W}=1$ domain walls are unique and very special in the sense that $\theta$ interpolates between one and the same physical vacuum state. Such $N_{D W}=1$ domain walls can be formed even if the PQ phase transition occurred before inflation and a unique physical vacuum occupies entire Universe.

It has been realized many years after the original publication [25] that the axion domain walls, in general, demonstrate a sandwich-like substructure on the QCD scale $\Lambda_{Q C D}^{-1} \simeq \mathrm{fm}$. The arguments supporting the QCD scale substructure inside the axion domain walls are based on analysis [26] of QCD in the large $N$ limit with inclusion of the $\eta^{\prime}$ field. It is also supported by analysis [27] of supersymmetric models where a similar $\theta$ vacuum structure occurs. The same structure also occurs in CS phase where the corresponding domain walls have been explicitly constructed [28].

\subsection{Spontaneous symmetry breaking of the baryon charge}

Second important element is that in addition to this known QCD substructures [26-28] of the axion domain walls expressed in terms of the $\eta^{\prime}$ and gluon fields, there is another substructure with a similar QCD scale which carries the baryon charge. Precisely this novel feature of the domain walls which was not explored previously in the literature will play a key role in our proposal because exactly this new effect will be eventually responsible for the accretion of the baryon charge by the nuggets. Both, the quarks and anti-quarks can accrete on a given closed domain wall making eventually the quark nuggets or anti-nuggets, depending on the sign of the baryon charge. The sign is chosen randomly such that equal number of quark and antiquark nuggets are formed if the external environment is $\mathrm{CP}$ even, which is the case when fundamental $\theta=0$.

Indeed, in the background of the domain wall, the physics essentially depends on two variables, $(t, z)$. One can show that in this circumstances the induced baryon charge $N$ for a single fermion in the axion domain wall background may assume any integer value $N$, positive or negative. The total baryon charge $B$ accumulated on a nugget is determined by the degeneracy factor in vicinity of the domain wall [1]

$$
N=\int d^{3} x \bar{\Psi} \gamma_{0} \Psi=-\left(n_{1}+n_{2}\right), \quad B=N \cdot g \cdot \int \frac{d^{2} x_{\perp} d^{2} k_{\perp}}{(2 \pi)^{2}} \frac{1}{\exp \left(\frac{\epsilon-\mu}{T}\right)+1}
$$

In this formula $g$ is appropriate degeneracy factor, e.g. $g \simeq N_{c} N_{f}$ in CS phase and $\mu$ is the chemical potential in vicinity of the domain wall. 
The main point of this section is that the domain walls generically will acquire the baryon or anti-baryon charge. This is because the domain wall tension is mainly determined by the axion field while the QCD substructure leads to small correction factor of order $\sim \Lambda_{\mathrm{QCD}} / f_{a} \ll 1$. Therefore, the presence of the QCD substructure with non vanishing $N \neq 0$ increases the domain wall tension only slightly. Consequently, this implies that the domain closed bubbles carrying the baryon or anti baryon charge will be copiously produced during the phase transition as they are very generic configurations of the system. Furthermore, the baryon charge cannot leave the system during the time evolution as it is strongly bound to the wall due to the topological reasons. The corresponding binding energy per quark is order of $\mu$ and increases with time as shown in [1]. One can interpret this phenomenon as a local spontaneous symmetry breaking effect, when on the scales of order the correlation length $\xi(T)$ the nuggets may acquire the positive or negative baryon charge with equal probability. This is because the sign of $N$ in eq. (5) may assume any positive or negative values with equal probabilities.

\subsection{Kibble-Zurek mechanism}

Next important ingredient of the proposal is the Kibble-Zurek mechanism which gives a generic picture of formation of the topological defects during a phase transition, see original papers [29], review [30] and the textbook [31]. In our context the Kibble-Zurek mechanism suggests that once the axion potential is sufficiently tilted the $N_{D W}=1$ domain walls form. The potential becomes much more pronounced when the chiral condensate forms at $T_{c}$. After some time after $T_{a}$ the system is dominated by a single, percolated, highly folded and crumpled domain wall network of very complicated topology. In addition, there will be a finite portion of the closed walls (bubbles) with typical size of order correlation length $\xi(T)$, which is defined as an average distance between folded domain walls at temperature $T$. It is known that the probability of finding closed walls with very large size $R \gg \xi$ is exponentially small.

The key point for our proposal is there existence of these finite closed bubbles made of the axion domain walls. Normally it is assumed that these closed bubbles collapse as a result of the domain wall pressure, and do not play any significant role in dynamics of the system. However, as we already mentioned in Introduction the collapse of these closed bubbles is halted due to the Fermi pressure acting inside of the bubbles. Therefore, they may survive and serve as the dark matter candidates. The percolated network of the domain walls will decay to the axion in conventional way as discussed in [32-35]. Those axions (along with the axions produced by the conventional misalignment mechanism $[33,36])$ will contribute to the dark matter density today. The corresponding contribution to dark matter density is highly sensitive to the axion mass as $\Omega_{\text {dark }} \sim m_{a}^{-1}$, and it is not subject of the present work. Instead, the focus of the present work is the dynamics of the closed bubbles, which is normally ignored in computations of the axion production. Precisely these closed bubbles, according to this proposal, will eventually become the stable nuggets and may serve as the dark matter candidates. The nugget's contribution to $\Omega_{\text {dark }}$ is not very sensitive to the axion mass, but rather, is determined by the formation temperature $T_{\text {form }}$ as explained in Introduction.

\subsection{Colour Superconductivity}

There existence of CS phase in QCD represents the next crucial element of our scenario. The CS has been an active area of research for quite sometime, see review papers [37, 38] on the subject. The CS phase is realized when quarks are squeezed to the density which is few times nuclear density. It has been known that this regime may be realized in nature in neutron stars interiors and in the violent events associated with collapse of massive stars or collisions of neutron stars, so it is important for astrophysics. 
The force which squeezes quarks in neutron stars is gravity; the force which does an analogous job in early universe during the QCD phase transition is a violent collapse of a bubble of size $R \sim \xi(T)$ formed from the axion domain wall as described in Section 3.3 above. If number density of quarks trapped inside of the bubble (in the bulk) is sufficiently large, the collapse stops due to the internal Fermi pressure. In this case the system starts to oscillate and the quarks in the bulk may reach the equilibrium with the ground state being in a CS phase. As we advocate in [1] this is very plausible fate of a relatively large size bubbles of size $R \sim \xi(T)$ made of the axion domain walls which were produced after the QCD phase transition.

Indeed, one can numerically solve the equations describing the evolution of the nuggets. A typical solution describes an oscillating bubble with frequency $\omega \sim m_{a}$. The bubble is slowly decreasing its radius $R(t)$ with a characteristic dumping scale $\tau$. The time evolution of such a bubble can be well approximated as follows

$$
R(t)=R_{\text {form }}+\left(R_{0}-R_{\text {form }}\right) e^{-t / \tau} \cos \omega t,
$$

where $R_{0}$ is initial size of a bubble $R_{0} \sim \xi(T)$, while $R_{\text {form }}$ is a final bible's size when formation is almost complete. In formula (6) parameter $\tau$ represents a typical damping time- scale which is expressed in terms of the axion mass $m_{a}$ and $\Lambda_{\mathrm{QCD}}$. It turns out that numerically $\tau$ is of order of cosmological scale $\tau \sim 10^{-4} \mathrm{~s}$. This numerical value is fully consistent with our anticipation that the temperature of the Universe drops approximately by a factor of $\sim 3$ or so during the formation period. During the same period of time the chemical potential $\mu$ inside the nugget reaches sufficiently large values when the CS sets in. Therefore, our phenomenological analysis in Section 2 (where DM nuggets are treated as very dense objects in CS phase) is supported by present studies on formation and time evolution of the nuggets made of the axion domain walls and ordinary quarks in CS phase.

\subsection{Coherent $C \mathcal{P}$ odd axion field}

If $\theta$ vanishes, then equal number of nuggets and anti-nuggets would form. However, the $C \mathcal{P}$ violating $\theta$ parameter (the axion field), which is defined as value of $\theta$ at the moment of domain wall formation generically is not zero, though it might be numerically quite small. Precisely the dynamics of the coherent axion field $\theta(t)$ leads to preferences in formation of one species of nuggets, as argued in details in [1]. This sign-preference is correlated on the scales where the axion field $\theta(t)$ is coherent, i.e. on the scale of the entire Universe at the moment of the domain wall formation. In other words, we assume that the PQ phase transition happened before inflation. One should emphasize that this assumption on coherence of the axion field on very large scales is consistent with formation of $N_{D W}=$ 1 domain walls, see section 3.1.

Precise dynamical computations of this $C \mathcal{P}$ asymmetry due to the coherent axion field $\theta(t)$ is a hard problem of strongly coupled QCD at $\theta \neq 0$. It depends on a number specific properties of the nuggets, their evolution, their environment, modification of the hadron spectrum at $\theta \neq 0$, etc. All these factors equally contribute to the difference between the nuggets and anti-nuggets. In order to effectively account for these coherent effects one can introduce an unknown coefficient $c(T)$ of order one as follows

$$
B_{\text {antinuggets }}=c(T) \cdot B_{\text {nuggets }}, \text { where }|c(T)| \sim 1,
$$

where $c(T)$ is obviously a negative parameter of order one. This key relation of this framework (7) unambiguously implies that the baryon charge in form of the visible matter can be also expressed in terms of the same coefficient $c(T) \sim 1$ as follows $B_{\text {visible }}=-\left(B_{\text {antinuggets }}+B_{\text {nuggets }}\right)$. Using eq. (7) the expression for the visible matter $B_{\text {visible }}$ can be rewritten as

$$
B_{\text {visible }} \equiv\left(B_{\text {baryons }}+B_{\text {antibaryons }}\right)=-[1+c(T)] B_{\text {nuggets }}=-\left[1+\frac{1}{c(T)}\right] B_{\text {antinuggets }}
$$


The same relation can be also represented in terms of the measured observables $\Omega_{\text {visible }}$ and $\Omega_{\text {dark }}$ at later times when only the baryons (and not anti-baryons) contribute to the visible component ${ }^{1}$

$$
\Omega_{\text {dark }} \simeq\left(\frac{1+|c(T)|}{|1+c(T)|}\right) \cdot \Omega_{\text {visible }} \text { at } T \leq T_{\text {form }} .
$$

One should emphasize that the relation (8) holds as long as the thermal equilibrium is maintained, which we assume to be the case. Another important comment is that each individual contribution $\left|B_{\text {baryons }}\right| \sim\left|B_{\text {antibaryons }}\right|$ entering (8) is many orders of magnitude greater than the baryon charge hidden in the form of the nuggets and anti-nuggets at earlier times when $T_{c}>T>T_{\text {form}}$. It is just their total baryon charge which is labeled as $B_{\text {visible }}$ and representing the net baryon charge of the visible matter is the same order of magnitude (at all times) as the net baryon charge hidden in the form of the nuggets and anti-nuggets.

The baryons continue to annihilate each other (as well as baryon charge hidden in the nuggets) until the temperature reaches $T_{\text {form }}$ when all visible anti-baryons get annihilated, while visible baryons remain in the system and represent the visible matter we observe today. It corresponds to $c\left(T_{\text {form }}\right) \simeq$ -1.5 as estimated below if one neglects the differences in gaps in CS and hadronic phases, see footnote 1. After this temperature the nuggets essentially assume their final form, and do not loose or gain much of the baryon charge from outside. The rare events of the annihilation between anti-nuggets and visible baryons continue to occur. In fact, the observational excess of radiation in different frequency bands, reviewed in section 2, is a result of these rare annihilation events at present time.

The generic consequence of this framework represented by eqs. (7), (8), (9) takes the following form for $c\left(T_{\text {form }}\right) \simeq-1.5$ which corresponds to the case when the nuggets saturate entire dark matter density today:

$$
B_{\text {visible }} \simeq \frac{1}{2} B_{\text {nuggets }} \simeq-\frac{1}{3} B_{\text {antinuggets }}, \quad \Omega_{\text {dark }} \simeq 5 \cdot \Omega_{\text {visible }}
$$

which is identically the same relation (2) presented in Introduction. The relation (10) emerges due to the fact that all components of matter, visible and dark, proportional to one and the same dimensional parameter $\Lambda_{\mathrm{QCD}}$, see footnote 1 with a comment on this approximation. In formula (10) $B_{\text {nuggets }}$ and

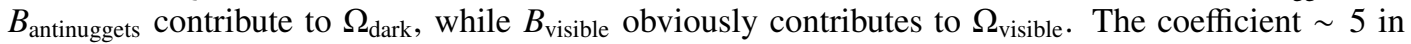

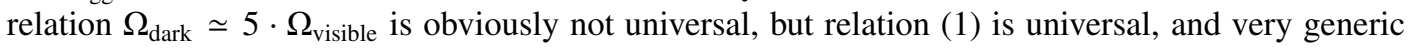
consequence of the entire framework, which was the main motivation for the proposal $[3,4]$.

\section{Implications for the axion search experiments}

The goal of this section is to comment on relation of our framework and the direct axion search experiments [8-15]. We start with the following comment we made in section 2: this model which has a single fundamental parameters (a mean baryon number of a nugget $\langle B\rangle \sim 10^{25}$ entering all the computations) is consistent with all known astrophysical, cosmological, satellite and ground based constraints as reviewed in section 2. For discussions of this section it is convenient to express this single normalization parameter $\langle B\rangle \sim 10^{25}$ in terms of the axion mass $m_{a} \sim 10^{-4} \mathrm{eV}$ as these two parameters directly related as discussed in the origin paper [3]. The corresponding relation between these two parameters emerges because the axion mass $m_{a}$ determines the wall tension $\sigma \sim m_{a}^{-1}$. At the same time parameter $\sigma$ enters the expression for equilibrium, which itself determines the size of

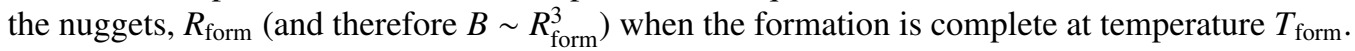

\footnotetext{
${ }^{1}$ In eq. (9) we neglect the differences (due to different gaps) between the energy per baryon charge in hadronic and CS phases to simplify notations. This correction obviously does not change the main claim of this proposal stating that $\Omega_{\text {visible }} \approx \Omega_{\text {dark }}$.
} 
The lower limit on the axion mass, as it is well known, is determined by the requirement that the axion contribution to the dark matter density does not exceed the observed value $\Omega_{\text {dark }} \approx 0.23$. There is a number of uncertainties in the corresponding estimates. We shall not comment on these subtleties by referring to the review papers [8-15]. The corresponding uncertainties are mostly due to the remaining discrepancies between different groups on the computations of the axion production rates due to the different mechanisms such as misalignment mechanism versus domain wall/string decays. If one assumes that the dominant contribution to the axion density is due to the misalignment mechanism than the estimates suggest that the axion of mass $m_{a} \simeq 2 \cdot 10^{-5} \mathrm{eV}$ saturates the dark matter density observed today, while the axion mass in the range of $m_{a} \geq 10^{-4} \mathrm{eV}$ contributes very little to the dark matter density.

There is another mechanism of the axion production when the Peccei-Quinn symmetry is broken after inflation. In this case the string-domain wall network produces a large number of axions such that the axion mass $m_{a} \simeq 10^{-4} \mathrm{eV}$ may saturate the dark matter density, see relatively recent estimates [33-35] with some comments and references on previous papers.

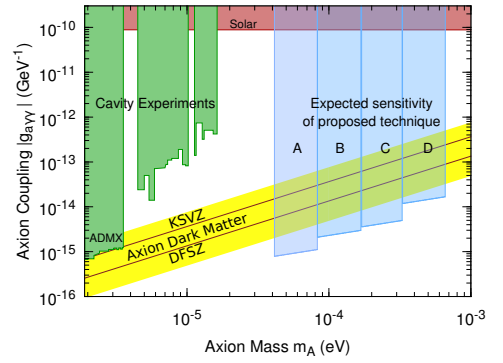

Figure 2. Cavity / ADMX experimental constraints on the axion mass shown in green. The expected sensitivity for the Orpheus axion search experiment [14] is shown by blue regions " $\mathrm{A}$ ", " $\mathrm{B}$ ", " $\mathrm{C}$ " and " $\mathrm{D}$ ". In particular, experiment " $\mathrm{B}$ ", covers the most interesting region of the parametrical space with $m_{a} \simeq 10^{-4} \mathrm{eV}$ corresponding to the nuggets with mean baryon charge $\langle B\rangle \simeq 10^{25}$ which itself satisfies all known astrophysical, cosmological, satellite and ground based constraints as discussed in section 2. The plot is taken from [14].

The main lesson to be learnt from the present work is that in addition to these well established mechanisms previously discussed in the literature there is an additional contribution to the dark matter density also related to the axion field. However, the mechanism which is advocated in the present work contributes to the dark matter density through formation of the nuggets, rather than through the direct axion production. The corresponding mechanism as argued in section 3.5 always satisfies the relation $\Omega_{\mathrm{dark}} \approx \Omega_{\mathrm{visible}}$, and, in principle is capable to saturate the dark matter density $\Omega_{\text {dark }} \approx 5 \Omega_{\text {visible }}$ by itself for arbitrary magnitude of the axion mass $m_{a}$ as the corresponding contribution is not sensitive to the axion mass in contrast with conventional mechanisms mentioned above. A precise coefficient in ratio $\Omega_{\mathrm{dark}} \approx \Omega_{\mathrm{visible}}$ is determined by a parameter of order one, $|c(T)| \sim 1$, which unfortunately is very hard to compute from the first principles, as discussed in section 3.5.

Our choice for $m_{a} \simeq 10^{-4} \mathrm{eV}$ which corresponds to $\langle B\rangle \sim 10^{25}$ is entirely motivated by our previous analysis of astrophysical, cosmological, satellite and ground based constraints as reviewed in Section 2. As we mentioned in Section 2 there is a number of frequency bands where some excess of emission was observed, and this model may explain some portion, or even entire excess of the observed radiation in these frequency bands. Our normalization $\langle B\rangle \sim 10^{25}$ was fixed by eq.(4) with assumption that the observed dark matter is saturated by the nuggets. The relaxing this assumption obviously modifies the coefficient $c(T)$ as well as $\langle B\rangle$.

Interestingly enough, this range of the axion mass $m_{a} \simeq 10^{-4} \mathrm{eV}$ is perfectly consistent with recent claim [39],[40] that the previously observed small signal in resonant $\mathrm{S} / \mathrm{N} / \mathrm{S}$ Josephson junction [41] is a result of the dark matter axions with the mass $m_{a} \simeq 1.1 \cdot 10^{-4} \mathrm{eV}$. We conclude this section on optimistic note with a remark that the most interesting region of the parametric space with mean 
baryon charge $\langle B\rangle \simeq 10^{25}$ which corresponds to $m_{a} \sim 10^{-4} \mathrm{eV}$ might be tested by the Orpheus axion search experiment [14] as shown on Fig. 2.

\section{Conclusion. Future directions.}

First, we want to list the main results of the present studies, while the comments on possible future developments will be presented at the end of this Conclusion.

1. First key element of this proposal is the observation (5) that the closed axion domain walls are copiously produced and generically will acquire the baryon or anti-baryon charge. This phenomenon of "separation of the baryon charge" can be interpreted as a local version of spontaneous symmetry breaking. This symmetry breaking occurs not in the entire volume of the system, but on the correlation length $\xi(T) \sim m_{a}^{-1}$ which is determined by the folded and crumpled axion domain wall during the formation stage. Precisely this local charge separation eventually leads to the formation of the nuggets and anti-nuggets serving in this framework as the dark matter component $\Omega_{\text {dark }}$.

2. Number density of nuggets and anti-nuggets and their size distributions will not be identically the same as a result of the coherent (on the scale of the Universe) axion $C \mathcal{P}$-odd field. We parameterize the corresponding effects of order one by phenomenological parameter $c(T) \sim 1$. It is important to emphasize that this parameter of order one is not fundamental constant of the theory, but, calculable from the first principles. In practice, however, such a computation could be quite a challenging problem when even the QCD phase diagram is not known. The fundamental consequence of this framework, $\Omega_{\text {dark }} \approx \Omega_{\text {visible, }}$, which is given by (1) is universal, and not sensitive to any parameters as both components are proportional to $\Lambda_{\mathrm{QCD}}$. The observed ratio (2), (10) corresponds to a specific value of $c\left(T_{\text {form }}\right) \simeq-1.5$ as discussed in section 3.5 .

3. Another consequence of the proposal is a natural explanation of the ratio (3) in terms of the formation temperature $T_{\text {form }} \simeq 40 \mathrm{MeV}$, rather than in terms of specific coupling constants which normally enter conventional "baryogenesis" computations. This observed ratio is expressed in our framework in terms of a single parameter $T_{\text {form }}$ when nuggets complete their formation. This parameter is not fundamental constant of the theory, and as such is calculable from the first principles. In practice, however, the computation of $T_{\text {form }}$ is quite a challenging problem as explained in the original paper [1]. Numerically, the observed ratio (3) corresponds to $T_{\text {form }} \simeq 40 \mathrm{MeV}$ which is indeed slightly below the critical temperature $T_{C S} \simeq 60 \mathrm{MeV}$ where the colour superconductivity sets in. The relation $T_{\text {form }} \lesssim T_{C S} \sim \Lambda_{\mathrm{QCD}}$ is universal in this framework as both parameters are proportional to $\Lambda_{\mathrm{QCD}}$. As such, the universality of this framework is similar to the universality $\Omega_{\text {dark }} \approx \Omega_{\text {visible }}$ mentioned in previous item. At the same time, the ratio (3) is not universal itself as it is exponentially sensitive to precise value of $T_{\text {form }}$ due to conventional suppression factor $\sim \exp \left(-m_{p} / T\right)$.

4. The only new fundamental parameter of this framework is the axion mass $m_{a}$. Most of our computations (related to the cosmological observations, see section 2.) however, are expressed in terms of the mean baryon number of nuggets $\langle B\rangle$ rather than in terms of the axion mass. However, these two parameters are unambiguously related as explained in the text.

5. This region of the axion mass $m_{a} \simeq 10^{-4} \mathrm{eV}$ corresponding to average size of the nuggets $\langle B\rangle \simeq 10^{25}$ can be tested in the Orpheus axion search experiment [14] as shown on Fig. 2.

We conclude with few thoughts on future directions within our framework. It is quite obvious that future progress cannot be made without a much deeper understanding of the QCD phase diagram at $\theta \neq 0$. In other words, we need to understand the structure of possible phases along the third dimension parametrized by $\theta$ on Fig 1 . Due to the known "sign problem", the conventional lattice simulations cannot be used at $\theta \neq 0$. Another possible development from the "wish list" is a deeper understanding of the closed bubble formation. Presently, very few results are available on this topic. 
The most relevant for our studies is the observation made in [10] that a small number of closed bubbles are indeed observed in numerical simulations. However, their detail properties (their fate, size distribution, etc) have not been studied yet. A number of related questions such as an estimation of correlation length $\xi(T)$, the generation of the structure inside the domain walls, the baryon charge accretion on the bubble, etc, hopefully can be also studied in such numerical simulations.

One more possible direction for future studies from the "wish list" is a development some QCDbased models where a number of hard questions such as: evolution of the nuggets, cooling rates, evaporation rates, annihilation rates, viscosity of the environment, transmission/reflection coefficients, etc in unfriendly environment with non-vanishing $T, \mu, \theta$ can be addressed, and hopefully answered. All these and many other effects are, in general, equally contribute to our parameters $T_{\text {form }}$ and $c(T)$ at the $\Lambda_{\mathrm{QCD}}$ scale in strongly coupled QCD. Precisely these numerical factors eventually determine the coefficients in the observed relations: $\Omega_{\text {dark }} \approx \Omega_{\text {visible }}$ given by eq. (9) and $\eta$ given by eq. (3).

Last but not least: the discovery of the axion in the Orpheus experiment [14] would conclude a long and fascinating journey of searches for this unique and amazing particle conjectured almost 40 years ago. Such a discovery would be a strong motivation for related searches of "something else" as the axion mass $m_{a} \simeq 10^{-4}$ is unlikely to saturate the dark matter density observed today. We advocate the idea that this "something else" is the "quark nuggets" (where the axion plays the key role in entire construction) which could provide the principle contribution to dark matter of the Universe as the relation $\Omega_{\text {dark }} \approx \Omega_{\text {visible }}$ in this framework is not sensitive to the axion mass.

\section{Acknowledgments}

This work was supported in part by the National Science and Engineering Research Council of Canada.

\section{References}

[1] X. Liang and A. Zhitnitsky, Phys. Rev. D 94, 083502 (2016) [arXiv:1606.00435 [hep-ph]].

[2] E. Witten, Phys. Rev. D 30, 272 (1984).

[3] A. R. Zhitnitsky, JCAP 0310, 010 (2003) [hep-ph/0202161].

[4] D. H. Oaknin and A. Zhitnitsky, Phys. Rev. D 71, 023519 (2005) [hep-ph/0309086].

[5] R. D. Peccei and H. R. Quinn, Phys. Rev. D 16, 1791 (1977);

S. Weinberg, Phys. Rev. Lett. 40, 223 (1978);

F. Wilczek, Phys. Rev. Lett. 40, 279 (1978).

[6] J.E. Kim, Phys. Rev. Lett. 43 (1979) 103;

M.A. Shifman, A.I. Vainshtein, and V.I. Zakharov, Nucl. Phys. B166 (1980) 493(KSVZ-axion).

[7] M. Dine, W. Fischler, and M. Srednicki, Phys. Lett. B104 (1981) 199;

A.R. Zhitnitsky, Yad.Fiz. 31 (1980) 497; Sov. J. Nucl. Phys. 31 (1980) 260 (DFSZ-axion).

[8] K. van Bibber and L. J. Rosenberg, Phys. Today 59N8, 30 (2006);

[9] S. J. Asztalos, L. J. Rosenberg, K. van Bibber, P. Sikivie, K. Zioutas, Ann. Rev. Nucl. Part. Sci. 56, 293-326 (2006).

[10] Pierre Sikivie, arXiv:0610440v2 [astro-ph].

[11] G. G. Raffelt, Lect. Notes Phys. 741, 51 (2008) [hep-ph/0611350].

[12] P. Sikivie, Int. J. Mod. Phys. A 25, 554 (2010) [arXiv:0909.0949 [hep-ph]].

[13] L. J. Rosenberg, Proc. Nat. Acad. Sci. (2015).

[14] G. Rybka, A. Wagner, A. Brill, K. Ramos, R. Percival and K. Patel, Phys. Rev. D 91, no. 1, 011701 (2015) [arXiv:1403.3121 [physics.ins-det]]. 
[15] P. W. Graham, I. G. Irastorza, S. K. Lamoreaux, A. Lindner and K. A. van Bibber, Ann. Rev. Nucl. Part. Sci. 65, 485 (2015) [arXiv:1602.00039 [hep-ex]].

[16] A. Zhitnitsky, Phys. Rev. D 74, 043515 (2006) [astro-ph/0603064].

[17] D. H. Oaknin and A. R. Zhitnitsky, Phys. Rev. Lett. 94, 101301 (2005), arXiv:hep-ph/0406146.

[18] A. Zhitnitsky, Phys. Rev. D 76, 103518 (2007), arXiv:astro-ph/0607361.

[19] K. Lawson and A. R. Zhitnitsky, JCAP 0801, 022 (2008), arXiv:0704.3064 [astro-ph].

[20] M. M. Forbes, K. Lawson and A. R. Zhitnitsky, Phys. Rev. D 82, 083510 (2010) [arXiv:0910.4541 [astro-ph.GA]].

[21] M. M. Forbes and A. R. Zhitnitsky, JCAP 0801, 023 (2008) [astro-ph/0611506].

[22] M. M. Forbes and A. R. Zhitnitsky, Phys. Rev. D 78, 083505 (2008) [arXiv:0802.3830 [astro$\mathrm{ph}]]$.

[23] K. Lawson and A. R. Zhitnitsky, Phys. Lett. B 724, 17 (2013) [arXiv:1210.2400 [astro-ph.CO]].

[24] K. Lawson and A. R. Zhitnitsky, Phys. Lett. B 757, 376 (2016) [arXiv:1510.02092 [astroph.GA]].

[25] P. Sikivie, Phys. Rev. Lett. 48 (1982) 1156;

A. Vilenkin and A.E. Everett, Phys. Rev. Lett. 48 (1982) 1867.

[26] M. M. Forbes and A. R. Zhitnitsky, JHEP 0110, 013 (2001) [arXiv:hep-ph/0008315].

[27] G. Gabadadze and M.A. Shifman, Phys. Rev.D 62(2000)114003 [arXiv:hep-ph/0007345].

[28] D. T. Son, M. A. Stephanov and A. R. Zhitnitsky, Phys. Rev. Lett. 86, 3955 (2001) [hep$\mathrm{ph} / 0012041]$.

[29] T.W.B. Kibble, J. Phys. A9, 1387 (1976);

W. Zurek, Nature 317, 505 (1985)

[30] W. Zurek, Phys. Rep. 276, 177 (1996)

[31] A. Vilenkin, E.P.S. Shellard, "Cosmic strings and other topological defects", Cambridge University Press, 1994

[32] S. Chang, C. Hagmann and P. Sikivie, Phys. Rev. D 59, 023505 (1999) [hep-ph/9807374].

[33] O. Wantz and E. P. S. Shellard, Phys. Rev. D 82, 123508 (2010) [arXiv:0910.1066 [astroph.CO]].

O. Wantz and E. P. S. Shellard, Nucl. Phys. B 829, 110 (2010) [arXiv:0908.0324 [hep-ph]].

[34] T. Hiramatsu, M. Kawasaki, K. Saikawa and T. Sekiguchi, Phys. Rev. D 85, 105020 (2012)

Erratum: [Phys. Rev. D 86, 089902 (2012)] [arXiv:1202.5851 [hep-ph]].

[35] M. Kawasaki, K. Saikawa and T. Sekiguchi, Phys. Rev. D 91, no. 6, 065014 (2015) [arXiv:1412.0789 [hep-ph]].

[36] J. Preskill, M. B. Wise, and F. Wilczek, Phys.Lett. B120, 127 (1983);

L. Abbott and P. Sikivie, Phys.Lett. B 120, 133 (1983);

M. Dine and W. Fischler, Phys.Lett. B 120, 137 (1983).

[37] M. G. Alford, A. Schmitt, K. Rajagopal and T. Schäfer, Rev. Mod. Phys. 80, 1455 (2008) [arXiv:0709.4635 [hep-ph]]

[38] K. Rajagopal and F. Wilczek, In *Shifman, M. (ed.): At the frontier of particle physics, vol. $3 *$ 2061-2151 [hep-ph/0011333].

[39] C. Beck, Phys. Rev. Lett. 111, 231801 (2013), [arxiv:1309.3790[hep-ph]];

[40] C. Beck, [arxiv:1403.5676 [hep-ph]]

[41] C. Hoffmann et al, Phys. Rev. B 70, 180503 (R) (2004) 